\title{
On quantum mechanical phase-space wave functions
}

\section{Wlodarz, Joachim J.}

Published in:

Journal of Chemical Physics

Link to article, DOI:

10.1063/1.466891

Publication date:

1994

\section{Document Version}

Publisher's PDF, also known as Version of record

Link back to DTU Orbit

Citation (APA):

Wlodarz, J. J. (1994). On quantum mechanical phase-space wave functions. Journal of Chemical Physics, 100(10), 7476-7480. https://doi.org/10.1063/1.466891

\section{General rights}

Copyright and moral rights for the publications made accessible in the public portal are retained by the authors and/or other copyright owners and it is a condition of accessing publications that users recognise and abide by the legal requirements associated with these rights.

- Users may download and print one copy of any publication from the public portal for the purpose of private study or research.

- You may not further distribute the material or use it for any profit-making activity or commercial gain

- You may freely distribute the URL identifying the publication in the public portal

If you believe that this document breaches copyright please contact us providing details, and we will remove access to the work immediately and investigate your claim. 


\section{On quantum mechanical phase-space wave functions}

Joachim J. Wlodarz

Department of Chemical Physics, Technical University of Denmark, DTII-301, DK-2800 Lyngby, Denmark and Department of Theoretical Chemistry, Silesian University, Szkolna 9, PL-40006 Katowice, Poland ${ }^{\text {a) }}$

(Received 4 October 1993; accepted 27 January 1994)

An approach to quantum mechanics based on the notion of a phase-space wave function is proposed within the Weyl-Wigner-Moyal representation. It is shown that the Schrödinger equation for the phase-space wave function is equivalent to the quantum Liouville equation for the Wigner distribution function. The relationship to the recent results by Torres-Vega and Frederick [J. Chem. Phys. 98, 3103 (1993)] is also discussed.

\section{INTRODUCTION}

Recently, a new phase-space representation of quantum mechanics has been proposed by Torres-Vega and Frederick. ${ }^{1}$ This representation is based on an abstract Hilbert space structure defined on the phase space in close analogy to the well-known Dirac formulation. ${ }^{2}$ The basic objects in this representation, the momentum $\hat{P}$ and position $\hat{Q}$ phase-space operators are chosen in the form

$$
\hat{P} \psi(p, q) \equiv\left(\gamma p+i \hbar \delta \frac{\partial}{\partial q}\right) \psi(p, q)
$$

and

$$
\hat{Q} \psi(p, q) \equiv\left(\alpha q+i \hbar \beta \frac{\partial}{\partial p}\right) \psi(p, q),
$$

where $\alpha, \beta, \gamma$, and $\delta$ arc some real constants. Taking into account the commutation relation between the position and momentum operators $[\hat{Q}, \hat{P}]=i \hbar \hat{I}$ and the various forms of the Liouville equation, the best values of these constants found in Ref. 1 were $\alpha=\gamma=1 / 2$ and $\beta=-\delta=1$. Other phasespace operators representing observable quantities were considered to be certain functions of these two basic operators. To avoid ordering problems, operators depending simultaneously on $\hat{Q}$ and $\hat{P}$ were not discussed. The quantum states are represented in this model by phase-space wave functions, evolving in time according to the phase-space Schrödinger equation.

In this paper we propose a similar approach, but based on the Weyl-Wigner-Moyal phase-space representation of quantum mechanics. ${ }^{3-5}$ We introduce a notion of phase-space wave function $\psi(p, q)$ which is directly related to the Wigner distribution function, and we show that the phasespace Schrödinger equation governing the time evolution of this wave function is equivalent to the quantum Liouville equation for the Wigner distribution function. The usage of the Weyl-Wigner-Moyal representation solves at the same time the operator ordering problems not addressed in Ref. 1. We show then that the quantity $|\psi(p, q)|^{2}$, a phase-space probability density, may be interpreted in some sense as the classical part of the Wigner distribution function. Comparing

a) Permanent address. our approach with that introduced in Ref. 1 we find that after suitable transformation of the momentum and position variables these both approaches coincide.

\section{THE WEYL-WIGNER-MOYAL FORMALISM}

The Weyl-Wigner-Moyal formulation of quantum mechanics is the first introduced and probably also the best known phase-space representation of quantum mechanics. In this approach the states of the system under consideration as well as the dynamical variables are represented through the appropriate phase-space functions according to the relation

$$
a^{w}(p, q)=h^{-N} \int d^{N} \tau \exp \left\{\frac{i}{\hbar} p \tau\right\}\left\langle q+\frac{\tau}{2}|\hat{A}| q-\frac{\tau}{2}\right\rangle
$$

known as the Weyl transform. ${ }^{6}$ Together with the $\star$ product of phase-space functions defined as follows: ${ }^{7}$

$$
\begin{aligned}
(a \star b)(p, q) \equiv & \left(\frac{2}{h}\right)^{2 N} \int d^{N} P_{1} d^{N} P_{2} d^{N} Q_{1} d^{N} Q_{2} \\
& \times \exp \left\{\frac{2 i}{\hbar}\left(Q_{1} P_{2}-Q_{2} P_{1}\right)\right\} \\
& \times a\left(P_{1}+p, Q_{1}+q\right) b\left(P_{2}+p, Q_{2}+q\right)
\end{aligned}
$$

it establishes an isomorphism between the algebra of operators of the standard quantum mechanics and the algebra of phase-space functions. Sometimes the * product is expressed in the differential form

$$
(a \star b)(p, q)=u(p, q) \exp \left\{\frac{\hbar}{2 i}\left(\frac{\grave{\partial}}{\partial p} \frac{\vec{\partial}}{\partial q}-\frac{\grave{\partial}}{\partial q} \frac{\vec{\partial}}{\partial p}\right)\right\} b(p, q),
$$

where the arrows indicate the function to be processed. It should be emphasized that these two forms are equivalent only when $a(p, q)$ and $b(p, q)$ are sufficiently regular phasespace functions. In general Eq. (2.3) is only an asymptotic expansion with respect to $\hbar$ of of the rhs of Eq. (2.2) (cf. Refs. 8-10 for more details).

The following relations involving the $\star$ product will be useful throughout this paper: 


$$
\begin{aligned}
& (a \star b)^{*}=b^{*} \star a^{*} \\
& \int_{\Gamma} d p d q(a \star b)(p, q)=\int_{\Gamma} d p d q a(p, q) b(p, q) \\
& \int_{\Gamma} d p d q a(p, q)(b \star c)(p, q) \\
& =\int_{\Gamma} d p d q b(p, q)(c \star a)(p, q) \\
& =\int_{\Gamma} d p d q c(p, q)(a \star b)(p, q)
\end{aligned}
$$

The states of a system are represented in the WeylWigner-Moyal representation through the famous Wigner distribution function ${ }^{4}$

$$
\rho^{w}(p, q)=h^{-N} \int d^{N} \tau \exp \left\{\frac{i}{\hbar} p \tau\right\}\left\langle q+\frac{\tau}{2}|\hat{\rho}| q-\frac{\tau}{2}\right\rangle
$$

which is the Weyl transform of the density operator $\hat{\rho}$.

It is known ${ }^{11}$ that the Weyl transform maps HilbertSchmidt operators onto square-integrable phase-space functions and vice versa. Because density operators are positive trace class operators which are also in the Hilbert-Schmidt class, the Wigner distribution functions are square integrable, too. Equipped with the scalar product in the usual form

$$
(f, g)=\int_{\Gamma} d p d q f^{*}(p, q) g(p, q)
$$

the space $L^{2}(\Gamma)$ of square-integrable phase-space functions becomes a Hilbert space.

Integration of a function over the whole phase space corresponds to taking a trace in the standard picture of quantum mechanics, and therefore the expectation value functional has here the following, classical-looking form

$$
\langle\not\rangle\rangle \equiv \operatorname{Tr}(\hat{A} \hat{\rho})=\int_{\Gamma} d p d q a^{w}(p, q) \rho^{w}(p, q) .
$$

There are many other known phase-space representations of quantum mechanics, but the Weyl-Wigner-Moyal representation occupies a unique position among them (see, e.g., Ref. 12 and references therein). Moreover, all of them are related to the Weyl-Wigner-Moyal representation. ${ }^{13}$

\section{WIGNER PHASE-SPACE WAVE FUNCTIONS}

It is well known (cf., e.g., Ref. 14) that any Hermitian trace class operator could be expressed as a product $\hat{A} \hat{A}^{\dagger}$, where $\hat{A}$ is an operator from the Hilbert-Schmidt class. As a corollary, any Wigner distribution function could be written in the form

$$
\rho^{w}(p, q)=\left(\psi \star \psi^{*}\right)(p, q),
$$

where $\psi \in L^{2}(\Gamma)$. The rôle played by $\psi(p, q)$ could be clearly seen after inserting the product $\psi \star \psi^{*}$ in the expectation value functional Eq. (2.9) instead of the Wigner distribution function $\rho^{\prime 1}(p, q)$ and rearranging the subintegral expression according to $\mathrm{Eq}$. (2.6):

$$
\begin{aligned}
\langle\mathscr{b}\rangle & =\int_{\Gamma} d p d p a^{w}(p, q)\left(\psi \star \psi^{*}\right)(p, q) \\
& =\int_{\Gamma} d p d p \psi^{*}(p, q)\left(a^{w} \star \psi\right)(p, q) .
\end{aligned}
$$

Introducing formally a (left) $\star$-product operator

$$
\breve{a}^{w^{w}} \psi(p, q) \equiv\left(a^{w} \star \psi\right)(p, q)
$$

we could write this expectation value functional in the familiar form

$$
\langle\mathscr{b}\rangle=\left(\psi, \breve{a}^{w} \psi\right)
$$

We see then that $\psi(p, q)$ plays exactly the same rôle as the ordinary wave function does in the standard formulation of quantum mechanics. Moreover, due to the properties of the $\star$ product [cf. Eq. (2.6)], all $\star$-product operators corresponding to physical observables are Hermitian.

All properties of ordinary wave functions in the position or momentum representation are valid also for these phasespace wave functions. For example, every phase-space wave function may be expressed as a linear combination of some mutually orthogonal basis elements, which could be furnished, e.g., by the set of eigenfunctions of a Hermitian phase-space operator

$$
\breve{a}^{w} \psi=\lambda \psi \text {. }
$$

Multiplying both sides of this equation by $\star \psi^{*}$ from the right and using Eq. (3.1) we get the eigenvalue equation for the Wigner distribution function

$$
\breve{a}^{w} \rho^{w}=\lambda \rho^{w}
$$

which has exactly the same form. However, Wigner distribution functions are real-valued while the phase-space wave functions are in general complex valued. Hence, when solving phase-space eigenvalue problems without imposing additional conditions we get always phase-space wave functions rather than Wigner distribution functions.

In the case of the phase-space Hamiltonian operator, i.e., when $\breve{a}^{w} \equiv \breve{h}^{w}$, we obtain the time-independent Schrödinger equation in its familiar form. This equation could be also easily derived from the general time-dependent Schrödinger equation (see next section).

Due to the definition of the $\star$ product these eigenvalue equations are in fact pseudodifferential equations (cf, e.g., Ref. 15) which are generally difficult to handle. As in the standard formulation of quantum mechanics, analytical solutions are possible only for the simplest systems, e.g., for the harmonical oscillator. To deal with other cases, appropriate approximative methods should be applied. Promising results were obtained recently by convertion of the eigenvalue problem Eq. (3.6) to a variational form. ${ }^{16,17}$

\section{THE PHASE-SPACE SCHRÖDINGER AND LIOUVILLE EQUATIONS}

Guided by the similarity to the standard approach to quantum mechanics, let us assume the time-dependent phase-space Schrödinger equation in the form 


$$
i \hbar \frac{\partial \psi_{t}}{\partial t}=\breve{h}^{w} \psi_{t} \equiv h^{w} \star \psi_{t} .
$$

Using the properties of the $\star$ product it could be easily shown that this phase-space Schrödinger equation is equivalent to the Liouville equation for the Wigner distribution function.

Let us write the complex conjugate of Eq. (4.1)

$$
i \hbar \frac{\partial \psi_{t}^{*}}{\partial t}=-\psi_{t}^{*} \star h^{w}
$$

Multiplying Eq. (4.1) by $\star \psi_{t}^{*}$ from the right and the Eq. (4.2) by $\psi_{t} \star$ from the left and adding the results side-by-side we get

$$
\begin{aligned}
i \hbar\left(\frac{\partial \psi_{t}}{\partial t} \star \psi_{t}^{*}+\psi_{t} \star \frac{\partial \psi_{t}^{*}}{\partial t}\right) & =h^{w} \star\left(\psi_{t} \star \psi_{t}^{*}\right) \\
& -\left(\psi_{t} \star \psi_{t}^{*}\right) \star h^{w}
\end{aligned}
$$

which, after insertion of $\rho_{t}^{w}$ for $\psi_{t} \star \psi_{t}^{*}$ gives the well-known quantum Liouville equation for the Wigner distribution function

$$
i \hbar \frac{\partial \rho_{t}^{w}}{\partial t}=h^{w} \star \rho_{t}^{w}-\rho_{t}^{w} \star h^{w} .
$$

The timc-indcpendent phase-space Schrödinger equation mentioned in the previous section could be also derived assuming the stationary phase-space wave function in the form

$$
\psi_{t}(p, q)=\exp \left\{-i \frac{\epsilon t}{\hbar}\right\} \psi(p, q) .
$$

Notice that the time-dependent phase factor will not appear in the corresponding. Wigner distribution function.

\section{INTERPRETATION OF THE WIGNER PHASE-SPACE WAVE FUNCTION}

It is well known that the Wigner distribution function, unlike the classical phase-space distribution functions, cannot be directly interpreted as a probability density. Various attempts have been undertaken to "smooth" the Wigner distribution function to get a probability density. Now it is known that the often postulated "coarse graining" approach by using phase-space cells of a finite size is impossible. ${ }^{18,19}$ Due to the Wigner theorem, ${ }^{20}$ we would sacrifice some useful properties when insisting on the positiveness of the quantum distribution function. Furthermore, using, e.g., the Husimi distribution function ${ }^{21}$ we even introduce some arbitrary parameters into the theory.

The phase-space wave function introduced above offers an additional possibility of getting phase-space probability densities, indicated also in Ref. 1. Namely, the quantity $|\psi(p, q)|^{2}$ as a real and everywhere nonegative phase-space function is a perfect candidate here. Moreover, as it will be shown below, it could be interpreted in some sense as the classical limit or, under certain limitations, even as a "classical part" of the Wigner distribution function.

Using the differential form of the $\star$ product [cf. Eq. (2.3)] we may write

$$
\rho^{w}=\psi \star \psi^{*}=\psi \psi^{*}+\mathscr{O}(\hbar)
$$

In the classical limit, when $\hbar \rightarrow 0$, the term $\mathscr{O}(\hbar)$ vanishes and $\rho^{w}(p, q)$ becomes equal to $|\psi(p, q)|^{2}$. In the case when the respective $\hbar$-expansion converges, we could split uniquely the $\star$ product into two parts

$$
(a \star b)(p, q)=a(p, q) b(p, q)+(a \diamond b)(p, q) .
$$

The first term $a(p, q) b(p, q)$ is the ordinary point-wise product of phase-space functions, while the remainder collecting all $\hbar$-depending terms forms the "diamond" product $(a \diamond b)(p, q)$. As before, in the limit when $\hbar \rightarrow 0$ we have that $(a \star b)(p, q) \rightarrow a(p, q) b(p, q)$, and therefore we may consider $a(p, q) b(p, q)$ as the "classical part" of the *-product and $(a \diamond b)(p, q)$ as the "quantum part." According to Eq. (5.2) we may then write the Wigner distribution function as

$$
\rho^{w}=\psi \star \psi^{*}=\psi \psi^{*}+\psi \diamond \psi^{*}
$$

and in this meaning $\psi \psi^{*}$ becomes the "classical part" of the Wigner distribution function. Moreover, we get then a oneone correspondence between the Wigner distribution function $\rho^{w}(p, q)$ and the respective phase-space probability density $|\psi(p, q)|^{2}$. According to Eq. (2.5) we have that

$$
\begin{aligned}
\int_{\Gamma} d p d q \rho^{w}(p, q) & =\int_{\Gamma} d p d q\left(\psi \star \psi^{*}\right)(p, q) \\
& =\int_{\Gamma} d p d q|\psi(p, q)|^{2}=1
\end{aligned}
$$

and also that

$$
\int_{\Gamma} d p d q\left(\psi \diamond \psi^{*}\right)(p, q)=0
$$

Hence, $|\psi(p, q)|^{2}$ is also always properly normalized to unity. Applying Eq. (5.2) to the rhs of Eq. (4.4) we find that the "classical" terms cancels each other and therefore the quantum Liouville equation may be also expressed in the "diamond" product form

$$
i \hbar \frac{\partial \rho^{w}}{\partial t}=h^{w} \diamond \rho^{w}-\rho^{w} \diamond h^{w} .
$$

It is interesting that $\psi(p, q)$ describes the state of a quantum system while at the same time $|\psi(p, q)|^{2}$ seems to describe its classical limit. This makes plausible to write the phase-space wave function in the factorized form

$$
\psi(p, q)=\operatorname{cxp}(i \varphi(p, q)) \psi_{C}(p, q),
$$

where $\psi_{C}(p, q) \equiv \sqrt{|\psi(p, q)|^{2}}$ is the "classical part" of the phase-space wave function and the "quantum part" appears as a complex phase factor.

Like in the approach introduced in Ref. 1, integration of the phase-space probability density $|\psi(p, q)|^{2}$ over one of the variables, $p$ or $q$, does not lead to the proper marginal densities in the position or momentum representations. However, an indirect link with these marginal densities is always provided by the respective Wigner distribution function, which integrated over $p$ or $q$ gives the correct marginal densities 


$$
\begin{aligned}
& \int_{\Gamma} d p \rho^{w}(p, q)=|\phi(q)|^{2} \\
& \int_{\Gamma} d q \rho^{w}(p, q)=|\varphi(p)|^{2} .
\end{aligned}
$$

\section{RELATION TO THE TORRES-VEGA AND FREDERICK APPROACH}

Now we will show that our approach and that proposed by Torres-Vega and Frederick are essentially the same when neglecting the operator ordering problems. This could be most conveniently done through comparing the phase-space Schrödinger equations introduced in both approaches.

The phase-space Schrödinger equation introduced in Ref. 1 has the form

$$
\begin{aligned}
i \hbar \frac{\partial}{\partial t}\left\langle p, q \mid \psi_{t}\right\rangle= & H\left[\left(\gamma p+i \hbar \delta \frac{\partial}{\partial q}\right),\left(\alpha q+i \hbar \beta \frac{\partial}{\partial p}\right)\right] \\
& \times\left\langle p, q \mid \psi_{t}\right\rangle
\end{aligned}
$$

which, after some algebra, could be rewritten to [cf. Eq. (2.25) in Ref. 1]

$$
\begin{aligned}
i \hbar \frac{\partial}{\partial t}\left\langle p, q \mid \psi_{t}\right\rangle= & H(\gamma p, \alpha q) \exp \left[i \hbar \delta \frac{\dot{\partial}}{\partial(\gamma p)} \frac{\vec{\partial}}{\partial q}\right. \\
& \left.+i \hbar \beta \frac{\dot{\partial}}{\partial(\alpha q)} \frac{\vec{\partial}}{\partial p}\right]\left\langle p, q \mid \psi_{t}\right\rangle .
\end{aligned}
$$

Notice that the momentum and position variables appearing in the Hamiltonian function are different from those appearing in the phase-space wave function. The differentiation operations are also performed in different ways for these two quantities.

In our approach, based on the Weyl-Wigner-Moyal phase-space representation, the observables and the states are treated exactly on the same footing and there is no difference in handling, e.g., the Hamiltonian function and the phasespace wave function.

Setting $\tilde{p}=\gamma p$ and $\tilde{q}=\alpha q$ as the common phase-space variables we see that Eq. (6.2) takes exactly the same form as Eq. (4.1) for $\alpha=\gamma=1 / 2$ and $\beta=-\delta=1$, the values chosen as canonical in Ref. 1 and assuming that the $\star$ product is expressed in the differential form.

The operators $\hat{P}$ and $\hat{Q}$ [see Eq. (1.1) and Eq. (1.2)] expressed in these common variables take in this case also the form of the Wigner operators introduced by Bopp ${ }^{22}$ and Kubo $^{23}$

$$
\begin{aligned}
& \hat{P}_{w}=\left(\tilde{p}-\frac{i \hbar}{2} \frac{\partial}{\partial \tilde{q}}\right), \\
& \hat{Q}_{w}=\left(\tilde{q}+\frac{i \hbar}{2} \frac{\partial}{\partial \tilde{p}}\right) .
\end{aligned}
$$

The "asymmetrical" treatment of observables and states may sometimes be more convenient than the "symmetrical" one. An important example is furnished here by the Fourier projection of phase-space wave functions onto the ordinary position or momentum wave functions introduced in Ref. 1 .
The idea of such projection consists in finding an intertwinning operator $\hat{T}_{q}$ (or $\hat{T}_{p}$ ) acting from $L^{2}(\Gamma)$ to $L^{2}(q)$ [or $\left.L^{2}(p)\right]$ and such that

$$
\hat{T}_{q} \hat{Q}_{\Gamma}^{n}=\hat{Q}_{q}^{n} \hat{T}_{q}
$$

and

$$
\hat{T}_{q} \hat{P}_{\Gamma}^{n}=\hat{P}_{q}^{n} \hat{T}_{q}
$$

holds for all $n \in \mathbb{N}$. The momentum and position operators are labeled here according to the respective functional spaces. This intertwinning operator enables us to convert the phase-space Schrödinger equation to its position (or momentum) equivalent, provided that all quantities appearing in this equation are analytic functions of position and/or momentum. The respective position (or momentum) wave function takes then simply the form

$$
\phi_{t}(q)=\hat{T}_{q} \psi_{t}(p, q)
$$

These intertwinning operators have a simple, Fouriertype integral transform form in the "asymmetric" TorresVega and Frederick approach [cf. Ref. 1], Eq. (4.19) and Eq. (4.23)]. Within our "symmetric" approach the appropriate intertwinning operators lose the Fourier-type character due to additional rescalings of the variables which need to be applied to the phase-space wave functions. In the "asymmetric" approach these rescalings are already included in the form of the phase space operators.

\section{SUMMARY}

The approach presented in this paper enables us to deal with quantum systems within the Weyl-Wigner-Moyal phase-space representation of quantum mechanics in the same manner as it is done in the standard picture of quantum mechanics. The phase-space Schrödinger equation being, as we have shown, equivalent to the quantum Liouville equation for the Wigner distribution function, is at the same time easier to handle from the applicational point of view. Although it is impossible to transform directly the phase-space wave functions to the appropriate wave functions in the position or momentum representation in the meaning of the Dirac transformation theory, all these wave functions and also the respective Wigner distribution function contain still the same information about the state of a system. It is so because the underlying algebraic structures are in all these approaches isomorphic. The quantity $|\psi(p, q)|^{2}$ could be interpreted as phase-space probability density and we show that it may be also regarded as the classical limit of the respective Wigner distribution function.

When limiting the considerations to the cases where operator orderings are not explicitly involved, the approach to the phase-space quantum mechanics proposed by TorresVega and Frederick ${ }^{1}$ differs from our only in the "asymmetric" treatment of observables and states. This asymmetry, although sometimes more useful on the quantum level, may cause difficulties in the classical limit. Our approach, being based on the Weyl-Wigner-Moyal formalism is free from this problem.

The author is deeply indebted to Professor Jens Peder Dahl for critical reading of the manuscript, several discus- 
sions and extremely warm hospitality during the stay in Lyngby. An acknowledgement is also made to the Commission of European Communities for the fellowship (ERB-CIPACT92-2096 8294) and to the Polish Committee for Scientific Research (KBN) for partial support.

${ }^{1}$ Go. Torres-Vega and J. H. Frederick, J. Chem. Phys. 98, 3103 (1993).

${ }^{2}$ P. A. M. Dirac, The Principles of Quantum Mechanics, 3rd ed. (Oxford University, Oxford, 1947).

${ }^{3}$ H. Weyl, Z. Phys. 46, 1 (1927).

${ }^{4}$ E. P. Wigner, Phys. Rev. 40, 749 (1932).

${ }^{5}$ J. E. Moyal, Proc. Camb. Phil. Soc. 45, 99 (1949).

${ }^{6}$ B. Leaf, J. Math. Phys. 9, 65 (1968).

${ }^{7}$ F. J. Narcowich and S. A. Fulling, Seminars in Mathematical Physics, No. 1 (Texas A\&M University, College Station, Texas, 1986).

${ }^{8}$ A. Voros, Ph.D. dissertation, Orsay, 1977.

${ }^{9}$ A. Voros, J. Funct. Anal. 29, 104 (1978).

${ }^{10}$ R. Estrada, J. M. Gracia-Bondia, and J. C. Varilly, J. Math. Phys. 30, 2789 (1989).
${ }^{11}$ J. C. T. Pool, J. Math. Phys. 7, 66 (1966).

${ }^{12}$ M. Hillery, R. F. O'Connell, M. O. Scully, and E. P. Wigner, Phys. Rep. 106, 121 (1984).

${ }^{13}$ G. C. Summerfield and P. F. Zweifel, J. Math. Phys. 10, 233 (1969).

${ }^{14}$ M. Reed and B. Simon, Methods of Modern Mathematical Physics, Vol. 1: Functional Analysis (Academic, New York, 1972).

${ }^{15} \mathrm{G}$. B. Folland, Harmonic Analysis in Phase Space (Princeton University, Princeton, NJ, 1989).

${ }^{16}$ J. J. Wlodarz and J. Nowakowski, Int. J. Quantum Chem. 38, 373 (1990).

${ }^{17} \mathrm{~J}$. J. Wlodarz (in press).

${ }^{18}$ J. J. Wlodarz, Phys. Lett. A 133, 459 (1988).

${ }^{19}$ D. M. Davidovic and D. Lalovic, in Classical and Quantum Systems: Foundations and Symmetries, edited by H. D. Doebner, W. Scherer, and F. Schroeck, Jr. (World Scientific, Singapore, 1993).

${ }^{20}$ E. P. Wigner, in Perspectives in Quantum Theory, edited by W. Yourgrau and A. Van der Merwe (MIT, Cambridge, 1971).

${ }^{21} \mathrm{~K}$. Husimi, Proc. Phys. Soc. Japan 22, 264 (1940).

${ }^{22} \mathrm{~F}$. Bopp, Werner Heisenberg und die Physik unserer Zeit (Vieweg Verlag, Braunschweig, 1961).

${ }^{23}$ R. Kubo, J. Phys. Soc. Jpn. 19, 2127 (1964). 